\title{
Phase Behavior in Thin Films of Confined Colloid-Polymer Mixtures
}

\author{
Chun-lai Ren and Yu-qiang Ma* \\ National Laboratory of Solid State Microstructures, Nanjing University, Nanjing 210093, China \\ Received November 6, 2005; E-mail: myqiang@nju.edu.cn.
}

\begin{abstract}
Using self-consistent field and density-functional theories, we first investigate colloidal selfassembling of colloid/polymer films confined between two soft surfaces grafted by polymers. With the increase of colloidal concentrations, the film undergoes a series of transitions from disordered liquid $\rightarrow$ sparse square $\rightarrow$ hexagonal (or mixed square-hexagonal) $\rightarrow$ dense square $\rightarrow$ cylindric structures in plane, which results from the competition between the entropic elasticity of polymer brushes and the steric packing effect of colloidal particles. A phase diagram displays the stable regions of different in-layer ordering structures as the colloidal concentration is varied and layering transitions as the polymer-grafted density is decreased. Our results show a new control mechanism to stabilize the ordering of structures within the films.
\end{abstract}

Keywords: Self-assembly, colloid-polymer films, self-consistent field theory; brushes; confinement

\section{Introduction}

Dispersions of colloidal particles in polymer solutions form the basic ingredients of a wide variety of systems which are essential to life and major industries $\stackrel{1,2}{\frac{1 n}{}}$ In particular, assembling colloidal nanoparticles into ordered structures 3.4 .5 .6 .7 .8 .9 .10 .11 .12 .13 .14 .15 .16 .17 .18 is important to the fabrication of nanomaterials with high performance in size-dependent optical, electrical, and mechanical properties. A major challenge in this field is to assemble monodispersed colloids into highly ordered structures with well-controlled sizes and shapes. Usually, the dispersed structures depend upon the effective interaction between the colloidal particles themselves and between the colloidal particles and their surroundings. For example, the interaction between colloidal particles in a solution of nonadsorbing polymers is influenced by the entropic excluded-volume (depletion) forces which were first recognized theoretically by Askura and Oosawa (AO) $\stackrel{19}{19}$ and thus a monodispersed colloidal system may typically crystallize into a close-packed structure or body-centered cubic lattice with high symmetry.

Thin films made of mixtures of polymer and colloidal particles can exhibit interesting structures, which are particularly useful for nano(bio)technology ranging from nanopatterning/lithography to changes of surface properties. Surface confinement modifies the bulk behavior of the system by breaking the symmetry of the structure, and therefore can be used to make low-dimensional ordering films. For nano-sized colloidal particles, the confinement of the suspension is a straightforward approach for the formation of colloidal crystals. For example, when the colloid/polymer mixtures are sandwiched between two parallel hard surfaces, layered structures may form, but the in-plane structure of the particles is always uniform or close-packed. Polymer brushes grafted to surfaces can modify the properties of the surface, ${ }^{20,21,22}$ which has many applications in colloidal stabilization and bio-compatibility. Interestingly, the grafted polymer chains may lead to a long-ranged repulsive force between the particles, which stabilizes the ordering of the nano-sized particles and controls the symmetry of the structure formed in a desired direction. Such a control is required for a wide range of applications using structuredependent optical, electrical, and mechanical properties of the colloidal crystals. It has been noted 23.24 .25 .26 .27 that thin films made of charged colloidal suspensions have various layered crystalline states if the film is confined between two parallel plates which repel the colloidal particles. Here, we show that for colloid/polymer mixtures, using polymer brush surfaces is an effective way of changing the in-plane structure of the colloidal film.

The purpose of the present article is to demonstrate a novel route of spatially organizing the colloid dispersions in polymer solutions confined between two polymergrafted plates. We find that with the deformation of grafted chains by the particles, the deformed brushes can exert an additional interaction between nano-sized colloidal particles, which stabilizes the formation of low symmetric ordering structures of colloids. Such a brushmediated colloidal effective interaction will compete with steric (center-force) effects of colloids, which may generate structures with more complicated lattice symmetries and undergo the symmetry-changing transitions in plane. In the present model, layering structures are formed due to confinement, and more importantly, the in-layer structural transitions are observed, depending upon the colloidal concentration and the polymer-grafted density. The results are summarized in a phase diagram displaying the stability regions of self-assembled ordering phases as functions of the colloidal concentration and the polymer-grafted density. The proposed approach is physically achievable for the generation of novel ordered structures. A successful example indicating the importance of such a self-assembling mechanism is the recent experimental verification ${ }^{10}$ of the predicated phase behavior of the copolymer/particle mixtures in confined geometries ${ }^{28.29}$ Another motivation of the present work is due to the fact that although many experiments have demonstrated the success of fabricating self-assembly nano-structures, the understanding of the physical mechanism that drives the self-assembly of the colloidal par- 
ticles in confined geometries is still lacking. $28,29,30,31$

\section{Model Details}

We consider a colloid suspension confined between two planar plates separated by a distance $L_{z}$ along the zaxis. The two plates which are grafted with $n_{b r}$ A-type homopolymer chains, are horizontally placed in $x y$-plane, and mechanically fixed at $z=0$ and $z=L_{z}$, respectively. All B-type free homopolymer chains in solutions are flexible with the same polymerization $N$ and statistical length $a$, and incompressible with a segment volume $\rho_{0}^{-1}$ as in A-type grafted chains. The volume of the system $V$ is $L_{x} \times \mathrm{E}_{y} \times L_{z}$, where $L_{x}$ and $L_{y}$ are the lateral lengthes of surfaces. The grafting density is defined as $\sigma=n_{b} /(2 \times$ $\left.L_{x} \times L_{y}\right)$; the average volume fraction of grafted chains is $\phi_{b}=n_{b} N \rho_{0}^{-1} / V$, the colloid $\phi_{c}=\left(1-\phi_{b}\right) \psi_{c}$ where $\psi_{c}$ is the colloidal concentration of the colloid-polymer mixture, and the free polymer $\phi_{p}=1-\phi_{b}-\phi_{c}$. Recently, the self-consistent field theory (SCFT) has been proven to be powerful in calculating equilibrium morphologies in polymeric systems, 32.33 .34 .35 .36 .37 .38 .39 .40 .41 .42 while colloidal particles can be treated by density-functional theory (DFT) $41,42,43,44$ to account for steric packing effects of particles. In our calculation, we use the hybrid SCFT/DFT approach developed in refs 41 and 42, and the free energy $\mathrm{F}$ for the present system is given by

$$
\begin{aligned}
\frac{N F}{\rho_{0} k_{B} T V}= & -\phi_{b} \ln \left(\frac{Q_{b}}{V \phi_{b}}\right)-\phi_{p} \ln \left(\frac{Q_{p}}{V \phi_{p}}\right)-\frac{\phi_{c}}{\alpha} \ln \left(\frac{Q_{c} \alpha}{V \phi_{c}}\right) \\
& +\frac{1}{V} \int d \mathbf{r}\left[\chi_{b p} N \varphi_{b}(\mathbf{r}) \varphi_{p}(\mathbf{r})+\chi_{b c} N \varphi_{b}(\mathbf{r}) \varphi_{c}(\mathbf{r})\right. \\
& +\chi_{p c} N \varphi_{p}(\mathbf{r}) \varphi_{c}(\mathbf{r})-W_{b}(\mathbf{r}) \varphi_{b}(\mathbf{r})-W_{p}(\mathbf{r}) \varphi_{p}(\mathbf{r}) \\
& -W_{c}(\mathbf{r}) \rho_{c}(\mathbf{r})-\xi(\mathbf{r})\left(1-\varphi_{b}(\mathbf{r})-\varphi_{p}(\mathbf{r})-\varphi_{c}(\mathbf{r})\right) \\
& \left.+\rho_{c} \Psi_{h s}\left(\bar{\varphi}_{c}\right)\right]
\end{aligned}
$$$$
+\chi_{p c} N \varphi_{p}(\mathbf{r}) \varphi_{c}(\mathbf{r})-W_{b}(\mathbf{r}) \varphi_{b}(\mathbf{r})-W_{p}(\mathbf{r}) \varphi_{p}(\mathbf{r}) \text { are in units of } a \text {. }
$$

where $k_{B}$ is the Boltzmann constant, and $T$ is the temperature. $\alpha$ is the volume ratio of the colloidal particle of radius $R$ to polymer chain: $\alpha=\frac{v_{R} \rho_{0}}{N}$, where $v_{R}=\frac{4}{3} \pi R^{3}$. $\varphi_{b}(\mathbf{r}), \varphi_{p}(\mathbf{r})$, and $\varphi_{c}(\mathbf{r})$ are the local volume fractions of brushes, free polymers, and colloids, respectively. $\xi(\mathbf{r})$ is the potential field that ensures the incompressibility of the system for dense colloid-polymer mixtures. $\rho_{c}$ stands for the particle center distribution, and the local particle volume fraction is then given by $\varphi_{c}(\mathbf{r})=$ $\frac{\alpha}{v_{R}} \int_{\left|\mathbf{r}^{\prime}\right|<R} d \mathbf{r}^{\prime} \rho_{c}\left(\mathbf{r}+\mathbf{r}^{\prime}\right) .41 .42$ The $\chi^{\prime}$ 's are the Flory interaction parameters between the different chemical species. $Q_{b}=\int d \mathbf{r} q_{1}(\mathbf{r}, s) q_{1}^{+}(\mathbf{r}, s)$ and $Q_{p}=\int d \mathbf{r} q_{2}(\mathbf{r}, 1)$ are single-chain partition functions for brushes and free polymers under the self-consistent fields $W_{b}(\mathbf{r})$ and $W_{p}(\mathbf{r})$, respectively. $Q_{c}=\int d \mathbf{r} \exp \left[-W_{c}(\mathbf{r})\right]$ is the partition function for colloids under the self-consistent field $W_{c}(\mathbf{r})$. The end-segment distribution functions $q_{i}(\mathbf{r}, s)$ and $q_{i}^{+}(\mathbf{r}, s)$ represent the probability of finding monomer s at position $\mathbf{r}$ respectively from two distinct ends of chains, which satisfy modified diffusion equations $\frac{\partial q_{i}}{\partial s}=\frac{a^{2} N}{6} \nabla^{2} q_{i}-$
$W_{i}(\mathbf{r}) q_{i}$, and $\frac{\partial q_{i}^{+}}{\partial s}=-\frac{a^{2} N}{6} \nabla^{2} q_{i}^{+}+W_{i}(\mathbf{r}) q_{i}^{+}$. For the grafted chains in the field $W_{b}(\mathbf{r})$, the initial condition is $q_{1}\left(x, y, z=0\right.$ or $\left.L_{z}, 0\right)=1, q_{1}\left(x, y, z \neq 0\right.$ or $\left.L_{z}, 0\right)=0$, and $q_{1}^{+}(x, y, z, 1)=1$, which means that the end of brush chains can move on the plates, although the total number of chains on surfaces is fixed. These have been called liquid brushes, in contrast to solid brushes where the immobile chains are anchored onto the surfaces. 45 Here we consider the liquid brush case because of its wide-ranging applications in colloidal and biological systems. For free polymers, the initial condition is $q_{2}(x, y, z, 0)=1$. The last term in Eq.(1) is DFT term ${ }^{43}$ accounting for the steric interaction between particles, and the excess free energy $\Psi\left(\bar{\varphi}_{c}\right)$ per particle is from the Carnahan-Starling function $^{44} \Psi_{h s}(x)=\frac{4 x-3 x^{2}}{(1-x)^{2}}$, with the weighted particle density $\bar{\varphi}_{c}(\mathbf{r}) \stackrel{41}{\stackrel{4}{\varphi}} \bar{\varphi}_{c}(\mathbf{r})=\frac{\alpha}{v_{2 R}} \int_{\left|\mathbf{r}^{\prime}\right|<2 R} d \mathbf{r}^{\prime} \rho_{c}\left(\mathbf{r}+\mathbf{r}^{\prime}\right)$, where $v_{2 R}$ is the volume of a sphere of radius $2 R$.

By minimizing the free energy in Eq. (1) with respect to $W_{b}(\mathbf{r}), W_{p}(\mathbf{r}), W_{c}(\mathbf{r}), \varphi_{b}(\mathbf{r}), \varphi_{p}(\mathbf{r}), \varphi_{c}(\mathbf{r})$, and $\xi(\mathbf{r})$, we obtain a set of self-consistent equations describing the equilibrium morphology of films, which can numerically be solved by the real space combinatorial screening algorithm of Drolet and Fredrickson.32.33 The free energy minimization of the system with respect to the selected simulation sizes is performed. ${ }^{46}$ We fix $N=100$, $L_{z}=50 a$, and the colloid diameter $2 R=0.6 R_{0}$, where $R_{0} \equiv a N^{\frac{1}{2}}$ characterizes the natural size of free polymers (typically $10 \sim 100 \mathrm{~nm}$ ). On the other hand, based on the assumption that the interaction between different chemical species should be small enough and polymer brushes are chemically neutral to free polymers and colloids, we choose the Flory-Huggins interaction parameters $\chi_{p c} N=5.0$ and $\chi_{b p} N=\chi_{b c} N=8.0$. All the sizes are in units of $a$.

\section{Results and Discussion}

We first explore the formation of ordered structures of colloidal crystallization in confined colloidal solution film. Figure 1 shows a series of particle-dispersed morphologies of varying the colloidal concentration $\psi_{c}$, and Figure 2 is the top views of profiles for polymer brushes grafted to one of the plates. We observe the formation of two layers of colloidal particles which are close to the polymer brush. When the colloidal concentration is small (for example, $\psi_{c}=0.29$, as in Figure 1(a)), colloids distribute uniformly in plane, and the brush-formed interfaces remain level(Figure 2(a)). With increasing the colloidal concentration, colloidal crystallization may appear, and inhomogeneous colloidal distribution will lead to the deformation of polymer brushes: caving where colloids exist and protruding where not. The deformed brushes prefer to stabilize the formation of low symmetric ordering structures of colloids, and with increasing the colloidal concentration, the particles grow anisotropically along one-dimensional direction. ${ }^{47}$ We see from 

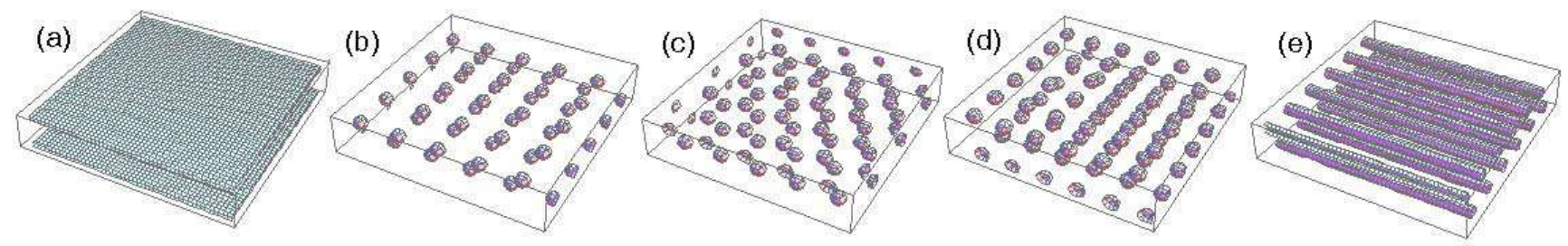

FIG. 1: Distributions of colloidal particles with increasing the colloidal concentration for the brush density $\sigma=0.2$ and $L_{x}=L_{y}=50$. The colloidal particles between two layers are alternatively packed each other. (a) $\psi_{c}=0.29,(\mathrm{~b}) \psi_{c}=0.30,(\mathrm{c})$ $\psi_{c}=0.35$, (d) $\psi_{c}=0.38$, and (e) $\psi_{c}=0.45$.
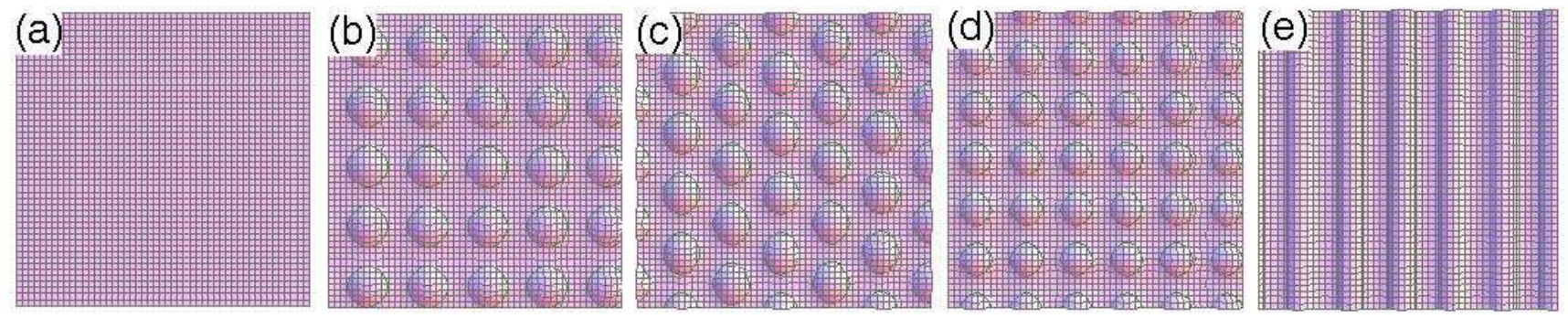

FIG. 2: Top views of the density profiles of polymer brushes. Parameters are the same as in Figure 1.

Figures 1(b)-1(e) that for different colloidal concentrations, colloids can assemble into sparse square, hexagonal, dense square, and cylindric structures, depending on the competition between the entropic effect of polymer brushes and steric packing effect of the particles. Figures 2(b)-2(e) show the corresponding shapes of deformed brush surface due to respective colloidal structures. When $\psi_{c}=0.30$, the average distance between colloidal particles is relatively large, and thus the brushmediated interaction between colloids dominates over the steric packing effect of colloids, leading to in-layer square structures for possibly large conformational entropy of polymer brushes(Figure 2(b)). When $\psi_{c}=0.35$, the stable state becomes hexagonal one(Figure 1(c) and 2(c)), indicating that colloidal steric packing effects dominate. However, a dense square structure appears at $\psi_{c}=0.38$ (Figure 1(d)), due to the requirement of entropic elastic release of deformed brushes. Further increase of colloids greatly limits the conformational space of brushes, and the entropy of brushes may be released by the formation of cylinder arrangement of colloids, because in this case, the free ends of grafted chains can at least move freely between parallel cylinders, as shown in Figure 1(e) and $2(\mathrm{e})$ when $\psi_{c}=0.45$. Figure 2 shows that the polymer brush is soft, and its density variation changes with the colloidal concentration. The range of the density variation in the direction perpendicular to the substrate is comparable to the size of the colloidal particles. The brush density close to the substrate remains unchanged with varying colloidal concentrations for a given grafting density.

Figure 3(a) shows the entropy of a grafted chain as a
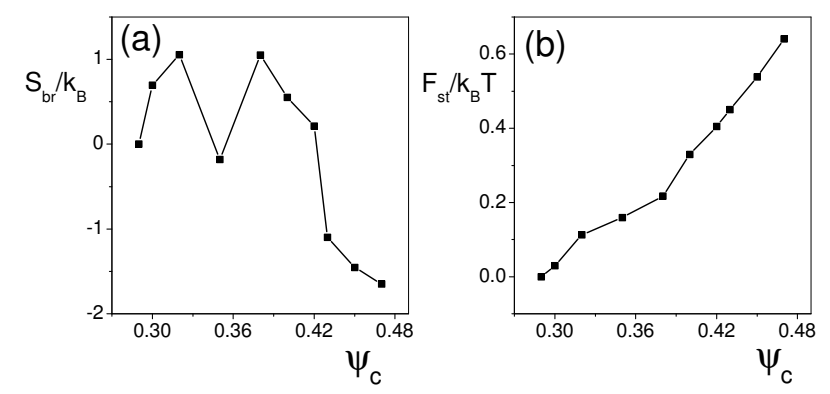

FIG. 3: (a) A grafted-chain entropy $\left(\frac{S_{b r}}{k_{B}}\right)$ vs the colloidal concentration; (b) the averaged steric energy per colloidal particle $\left(\frac{F_{s t}}{k_{B} T}\right)$ vs the colloidal concentration.

function of the colloidal concentration $\psi_{c}$. We set the grafted chain's entropy $\frac{S_{b r}}{k_{B}}=0$, when the dispersion of colloidal particles is uniform in plane $\left(\psi_{c}=0.29\right)$. The entropy of polymer brushes, with colloidal concentrations varied from $\psi_{c}=0.29$ to $\psi_{c}=0.32$, increases with the formation of colloidal square structures. However, the brush entropy curve suddenly decreases with the influence of steric interaction of colloids, and a hexagonal structure is formed when $\psi_{c}=0.35$. With further increase of colloidal concentrations, the deformed brushes begin to dominate over the steric energy of colloids again, which prefers to release the conformational space of brushes with the gradual formation of square structures. The brush entropy arrives at the maximum 
at $\psi_{c}=0.38$, corresponding to the formation of dense square lattice structure (Figure $1(\mathrm{~d})$ ). After that, brush entropy decreases with the increase of colloidal concentrations, and colloidal cylinder structures begin to form. The complete cylindric structure appears at $\psi_{c}=0.42$, and remains unchanged even for relatively larger colloidal concentrations. Usually, the entropic gain or loss due to the conformational change of polymer chains may signify the structural transitions of the polymeric system. For example, Balazs and co-workers ${ }^{41.42}$ studied an $\mathrm{AB}$ diblock-nanoparticle composite, in which the particles and diblock chains form spatially periodic structures. They observed a similar behavior of A-block entropic free energy when the volume fraction of the particles is increased. $\stackrel{41}{ }$ In their work, however, the conformational entropy of the A-block only shows the stretching and compressing of the A block chains along the chain direction, because they considered the formation of spatially periodic structures in two-dimensional copolymernanoparticle composites in the plane of the polymer chains. On the contrary, in the present three-dimensional confined mixtures of colloidal particles and free homopolymer chains, we emphasis a control mechanism in the lateral direction of the colloidal suspension driven by the deformed chains in the polymer brush. Actually, the entropic free energy in Figure 3(a) depicts the shape changes of brush surface (Figure 2) during the variation of the colloidal concentration for a given grafting density $\sigma=0.2$. The configurational entropy contribution of the grafted chains originates from the free ends of the brushes comparable in size to the colloidal particles, since the bottom part of the chains close to the substrate is strongly stretched and remains nearly unchanged with varying colloidal concentration for a fixed grafting density. This means that the entropic effect of the deformed brushes by the particles comes mainly from the lateral conformation fluctuations of the free chain ends, and that the averaged conformational entropy contribution of the polymer brushes along the chain-stretching direction can be ignored. Such an entropic effect competes with the steric packing effect of the particles in the plane, leading to structure changes in the plane of the colloidal particles. Here, a significant advantage of using soft polymer-grafted substrate is that the deformed brush provides an in-plane long-ranged anisotropic interaction between the colloidal particles. In Figure 3(b), the average steric energy per colloidal particle varies with the increase of colloidal concentration, which clearly reflects the excluded-volume effect of colloids. As a whole, steric energy increases with increasing the colloidal concentration $\psi_{c}$. Interestingly, we find that the slope of the curve has apparent difference in different ranges of $\psi_{c}$. Particularly in the region $\psi_{c} \sim 0.32-0.38$ where the morphology inclines to adopt hexagonal or mixed square-hexagonal structure, the slope is distinctly lower than that of other regions. This means that the steric energy can be released by forming hexagonal or mixed hexagonal/square structures.
Further, we also observe the appearance of layering transitions with the increase of effective film thickness via decreasing the height of polymer brushes. The effective thickness of film can be defined by subtracting the brush height from the distance $L_{z}$ between two substrates, i.e., $d_{e f f}=L_{z}-2 h$, where the dry brush height $h$ satisfies $h=\sigma N / \rho_{0}$ in the incompressible system of brush and homopolymer molecules ${ }^{48}$ Thus, the effective thickness $d_{\text {eff }}$ of the film is changeable, and increases with decreasing the polymer-grafted density $\sigma$. Figures 4(a)-4(c) show the possible three-layer structures such as mixed sphere-cylinder, complete cylinder, and even sphere structures for the selected values of $\sigma$ and $\psi_{c}$. To clarify the phase stability of different ordering structures,$\frac{49}{9}$ we calculate the phase diagram as functions of the grafting density and the colloidal concentration, as shown in Figure 5. Here, we choose the grafting density $\sigma$ within the range $[0.15,0.21]$ for two reasons: on the one hand, the entropic change of deformed brushes should be large enough to cause effective brush-mediated interaction between colloids, requiring that the value of $\sigma$ can not be too small; on the other hand, the effective film thickness should be thick enough (namely $\sigma$ is reasonably small) to ensure the possibility of colloidal crystallization. We see from Figure 5 that when the grafting density is relatively large, the two-layered colloidal structure undergoes disordered liquid, square, and cylinder ones. In this case, the brush-mediated interaction always dominates, which drives the self-assembly of colloids into square lattice at small $\psi_{c}$ and cylinder structure at larger $\psi_{c}$. The hexagonal phase disappears at the middle concentrations of colloids. For intermediate values of polymer-grafted densities $(\sigma=0.21 \sim 0.18)$, two-layered structure remains unchanged, but a reentrant structure transition between sparse square $\rightarrow$ hexagonal (or squarehexagonal) $\rightarrow$ dense square lattice structures appears as a result of the competition between brush-mediated interaction and steric packing effects of colloids. When $\sigma$ is less than 0.18 , layering transition may appear for sufficiently large values of $\psi_{c}$. The three-layered spherecylinder structure $\left(C S_{3}\right.$ phase $)$ first appears, where cylinder structures closing to brush surfaces are formed because of colloidal particles favoring the aggregation onto brush surfaces at first, and the middle layer still retains discrete spherical structure. With increasing the colloidal concentration, the cylinder-forming monolayer becomes a template which drives the ordering of other colloidal particles in the middle of the film with the help of the deformation of free polymers in the suspension, and then the three-layered cylinder structure $\left(C_{3}\right)$ appears. If $\sigma$ is decreased below 0.165 , three-layered spherical structure $\left(S_{3}\right.$ phase) may be formed for intermediate ranges of $\psi_{c}$, before the appearance of mixed sphere-cylinder structures. The phase diagram shown in Fig. 5 also provides useful information about other structural changes of the system. For example, one can observe the structural and layering transitions with increasing effective film thickness (equivalently expanding the distance between the two plates) 

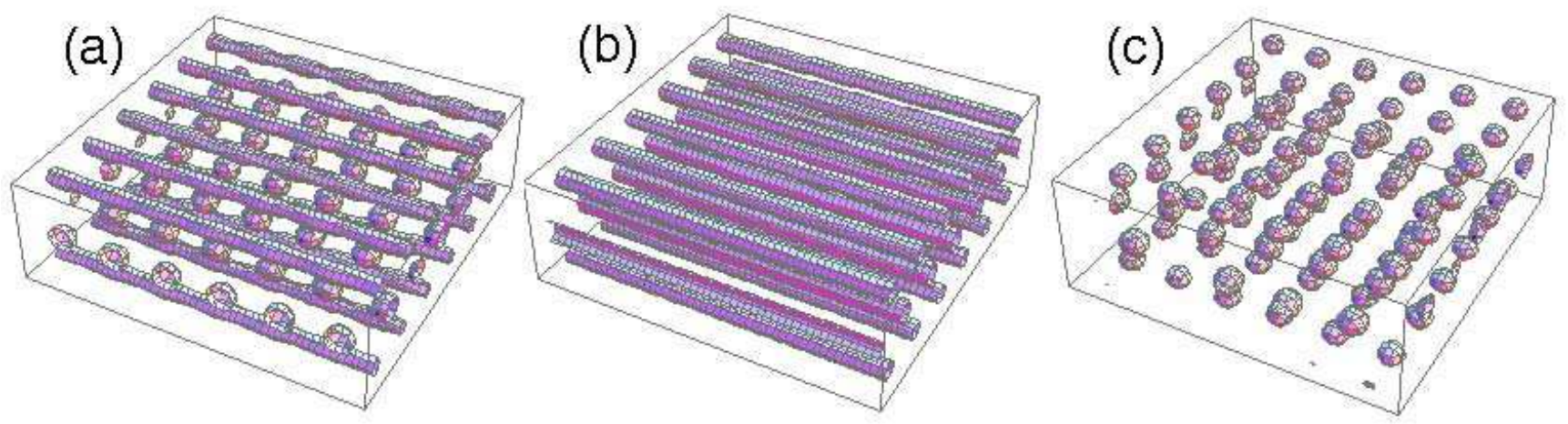

FIG. 4: The formation of three-layer structures: (a) sphere-cylinder mixture when $\sigma=0.17$ and $\psi_{c}=0.36$; (b) cylinder structure when $\sigma=0.17$ and $\psi_{c}=0.42$; (c) spherical structure when $\sigma=0.16$ and $\psi_{c}=0.32$.

\section{Conclusions}

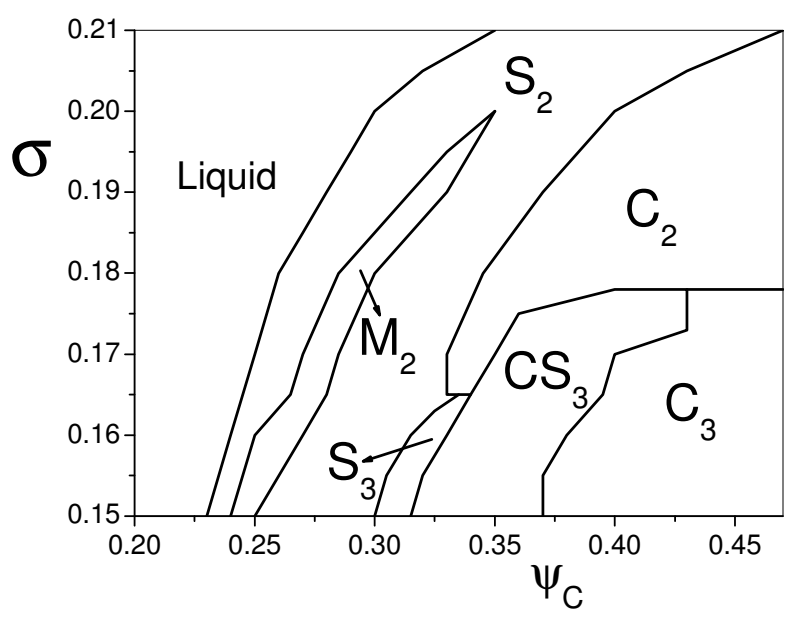

FIG. 5: The phase diagram as a function of the grafting density and the colloidal concentration. $S_{2}$ : two-layered sparse or dense square structure; $M_{2}$ : two-layered hexagonal or mixed square-hexagonal structure; $C_{2}$ : two-layered cylindric structure; $S_{3}$ : three-layered spherical structure; $C S_{3}$ : threelayered cylindric-spherical mixed structure; $C_{3}$ : three-layered cylindric structure.

via decreasing grafting density $\sigma$ for fixed colloidal concentration $\psi_{c}$. If one changes the distance between the two plates while increasing the colloidal concentration, the colloidal structural transitions can be obtained along a tilted direction from the left to right in Figure 5.
We study the phase behavior in thin films of confined colloid/polymer mixtures. Depending on the competition between brush-mediated interaction and steric packing effects of colloids, the colloidal self-assembly can experience a series of in-layer symmetry-changing transitions between square lattice, hexagonal, and cylinder structures, which can be induced by changing the colloidal concentration. Furthermore, the layering transitions can be obtained by changing the film thickness of the system. We find that the colloidal particles lying on the polymer brush form a monolayer, in which the deformed chains produce an in-plane long-ranged anisotropic interaction, which controls the ordering of the colloidal particles. This layer of ordered particles then becomes a template on which the other colloidal particles in the middle of the film grow into a large ordered structure with the help of free polymers in the region. The results may provide a helpful guide for fabricating the functionally useful microstructures in materials science of complex liquids. The suggested approach is also suitable to understanding colloidal self-assembling on the other soft walls such as the packing of biomacromolecules confined within the bio-membranes.

\section{Acknowledgment}

This work was supported by the National Natural Science Foundation of China, No. 10334020, No. 10021001, No. 20490220, and No. 10574061.
1 Russel, W. B.; Saville, D. A.; Schowalter, W. R. Colloidal Dispersions, Cambridge University Press, Cambridge 1989.

2 Fuchs, M.; Schweizer, K. S. J. Phys.: Condens. Matt. 2002, 14, R239.
3 Whitesides, G. W.; Boncheva, M. Proc. Natl. Acad. Sci. 2002, 99, 4769.

4 Liu, Z.; Pappacena, K.; Cerise, J.; Kim, J.; Durning, C. J.; O'Shaughnessy, B.; Levicky, R. Nano. Lett. 2002, 2, 219.

${ }^{5}$ Zhu, J.; Li, M.; Rogers, R.; Meyer, W.; Ottewill, R. H.; 
STS-73 Space Shuttle Crew, Russel, W. B.; Chaikin, P. M. Nature 1997, 38\%, 883.

6 van Blaaderen, A. ; Ruel, R.; Wiltzius, P. Nature 1997, 385, 321.

7 Yethiraj, A.; van Blaaderen, A. Nature 2003, 421, 513.

8 Ramos, L.; Lubensky, T. C.; Dan, N.; Nelson, P.; Weitz, D. A. Science 1999, 286, 2325.

9 Lin, K. H.; Crocker, J. C.; Prasad, V.; Schofield, A.; Weitz, D. A.; Lubensky, T. C.; Yodh, A. G. Phys. Rev. Lett. 2000, 85,1770

10 Lin, Y.; Boker, A.; He, J.B.; Sill, K.; Xiang, H. Q.; Abetz, C.; Li, X. F.; Wang, J.; Emrick, T.; Long, S.; Wang, Q.; Balazs, A. C.; Russell, T. P. Nature 2005, 434, 55.

11 Lin, Y.; Skaff, H.; Emrick, T.; Dinsmore, A. D.; Russell, T. P. Science 2003, 299, 226.

12 Bechinger, C. Curr. Opin. Colloid Interface Sci. 2002, 7, 204.

13 Wang, D. Y.; Mohwald, H. J. Mater. Chem. 2004, 14, 459.

14 Xia, Y.; Yin, Y.; Lu, Y.; Mclellan, J Adv. Funct. Mater. 2002, 14, 605.

15 Yin, Y.; Lu, Y.; Gates, B.; Xia, Y. J. Am. Chem. Soc. 2001, 123, 8718.

16 Yin, Y.; Xia, Y. Adv. Mater. 2002, 14, 605.

17 Kim, E.; Xia, Y.; Whitesides, G. M. Adv. Mater. 1996, 8, 245.

18 Yang, P.; Rivzi, A. H.; Messer, B.; Chemlka, B. F.; Whitesides, G. M.; Stucky, G. D. Adv. Mater. 2001, $13,427$.

19 Asakura, S.; Oosawa, F. J. Polym. Sci. 1958, 33, 183.

20 Milner, S. T. Science 1991, 251, 905.

21 (a) Satulovsky, J.; Carignano, M. A.; Szleifer, I. Proc. Natl. Acad. Sci. 2000, 97, 9037. (b) Currie E. P. K.; Norde W.; Cohen Stuart, M. A. Adv. Coll. Inter. Sci. 2003, 100-102, 205.

22 Ren, C. L.; Chen, K.; Ma, Y. Q. J. Chem. Phys. 2005, 122, 154904.

23 Murray, C. A.; D. G. Grier, Annu. Rev. Phys. Chem. 1996, $4 \%, 421$.

${ }^{24}$ Messina, R.; Löwen, H. Phys. Rev. Lett. 2003, 91, 146101.

25 Schmidt, M.; Löwen, H. Phys. Rev. Lett. 1996, 24, 4552.

26 Wasan, D. T.; Nikolov, A. D. Nature 2003, 423, 156.

27 Henderson, D.; Trokhymchuk, A.; Nikolov, A.; Wasan, D. T. Ind. Eng. Chem. Res. 2005, 44, 1175.

${ }^{28}$ Lee, J. Y.; Shou, Z.; Balazs, A. C. Phys. Rev. Lett. 2003,
91, 136103.

29 Lee, J. Y.; Shou, Z.; Balazs, A. C. Macromolecules 2003, 36,7730

${ }^{30}$ Lee, J. Y.; Buxton, G. A.; Balazs, A. C. J. Chem. Phys. 2004, 121, 5531.

31 Buxton, G. A.; Lee, J. Y.; Balazs, A. C. Macromolecules 2003, 36, 9631.

32 Drolet, F.; Fredrickson, G. H. Phys. Rev. Lett. 1999, 83, 4317.

33 Drolet, F.; Fredrickson, G. H. Macromolecules 2001, 34, 5317.

34 Schmid, F. J. Phys.: Condens. Matter 1998, 10, 8105.

35 Matsen, M. W.; Schick, M. Phys. Rev. Lett. 1994, 72, 2660.

36 Petera, D.; Muthukumar, M. J. Chem. Phys. 1998, 109, 5101.

37 Matsen, M. W.; Bates, F. S. J. Chem. Phys. 1997, 106, 2436.

38 Maniadis, P.; Thompson, R. B.; Rasmussen, K.; Lookman, T. Phys. Rev. E 2004, 69, 031801.

39 Muller, M. Phys. Rev. E 2002, 65, 030802.

40 Geisinger, T.; Muller, M.; Binder, K. J. Chem. Phys. 1999, $111,5241$.

41 Thompson, R. B.; Ginzburg, V. V.; Matsen, M. W.; Balazs, A. C. Science 2001, 292, 2469.

42 Thompson, R. B.; Ginzburg, V. V.; Matsen, M. W.; Balazs, A. C. Macromolecules 2002, 35, 1060.

43 Tarazona, P. Mol. Phys. 1984, 52, 81.

${ }^{44}$ Carnahan, N. F.; Starling, K. E. J. Chem. Phys. 1969, 51, 635.

45 Subramanian, G.; Williams, D. R. M.; Pincus, P. A. Macromolecules 1996, 29, 4045.

46 Bohbot-Raviv, Y.; Wang, Z. G. Phys. Rev. Lett. 2000, 85, 3428.

47 Kim, J. U.; O'Shaughnessy, B. Phys. Rev. Lett. 2002, 89, 238301.

48 Matsen, M. W. J. Chem. Phys. 2005, 122, 144904.

49 To search the stable phases of the film, we first ascertain the symmetries of possible structure formation, and then calculate the free energy of the film. Furthermore, the total free energy minimization of the system with respect to the selected simulation sizes is required by adjusting the lateral dimension ${ }^{46}$. 